\title{
On convolution surfaces in Euclidean 3-space
}

\author{
Selin Aydöner ${ }^{\mathrm{a}}$ and Kadri Arslan ${ }^{\mathrm{b} *}$ \\ ${ }^{a}$ Department of Mathematics, Institute of Natural and Applied Science, Uludă̆ University, Bursa, Turkey \\ ${ }^{\mathrm{b}}$ Department of Mathematics, Faculty of Science and Arts, Uluda ğ University, Bursa, Turkey \\ *Corresponding author E-mail: arslan@uludag.edu.tr
}

\section{Article Info}

Keywords: Convolution of surfaces, Flat surfaces, Gaussian curvature, Minkowski sum, Second fundamental form

2010 AMS: $53 C 40,53 C 42$

Received: 18 May 2018

Accepted: 24 September 2018

Available online: 30 September 2018

\begin{abstract}
In the present paper we study with the convolution surface $C=M \star N$ of a paraboloid $M \subset \mathbb{E}^{3}$ and a parametric surface $N \subset \mathbb{E}^{3}$. We take some spacial surfaces for $N$ such as, surface of revolution, Monge patch and ruled surface and calculate the Gaussian curvature of the convolution surface $C$. Further, we give necessary and sufficient conditions for a convolution surface $C$ to become flat.
\end{abstract}

\section{Introduction}

Given two objects $A$ and $B$ in $\mathbb{R}^{3}$, their Minkowski sum $A \oplus B$ is defined to be the set

$$
A \oplus B:=\{a+b: a \in A, b \in B\}
$$

where $a$ and $b$ denote position vectors of arbitrary points in $A$ and $B$. Minkowski sums in two and three dimensions are used in various fields, for example mathematical morphology, computer graphics, convex geometry, computational geometry motion planning. The algorithmic problem for polynomial and polyhedral shapes as well as approximations of the convolution and Minkowski sum have been studied, see for instance ([3], [5]) and the references therein. Let $M=\partial A$ and $N=\partial B$ be boundaries of $A$ and $B$ respectively. Then, the computation of the boundary $\partial(A \oplus B)$ is related to the computation of the convolution surface $M \star N$ of the two boundary surfaces $M$ and $N$. We always assume in the following that $M$ and $N$ are smooth surfaces with normal vector fields $\overrightarrow{n_{M}}$ and $\overrightarrow{n_{N}}$, respectively. The convolution surface is defined to be

$$
M \star N:=\left\{x+y: x \in M, y \in N, \text { and } \overrightarrow{n_{M}} \| \overrightarrow{n_{N}}\right\}
$$

where $\overrightarrow{n_{M}}(x)$ and $\overrightarrow{n_{N}}(y)$ are mutually parallel normal vectors at points $x$ and $y([4]$, [2] ). In particular, if $A$ and $B$ are convex objects, the boundary $\partial(A \oplus B)$ of the Minkowski sum $A \oplus B$ is exactly given by the convolution surface $M \star N$. Unfortunately, for non-convex objects this property is no longer true. In general, the boundary $\partial(A \oplus B)$ of the Minkowski sum is contained in the convolution surface $M \star N$, formed by the boundaries $M=\partial A$ and $N=\partial B$, respectively. The boundary $\partial(A \oplus B)$ of the Minkowski sum $A \oplus B$ is contained in the envelope of $B$ with respect to the translations $x^{\prime}=a+x, a \in A$ (see, [1]).

In general, the computation of the convolution surface $M \star N$ of two smooth surfaces $M$ and $N$ results in the following way. Assume that the surfaces $M$ and $N$ are parametrized by $x=x(u, v)$ and $y=y(s, t)$, respectively and that the normal vectors are denoted by $\overrightarrow{n_{M}}(u, v)$ and $\overrightarrow{n_{N}}(s, t)$. The convolution surface $M \star N$ is formed by the sums of the position vectors $x, y$ of the surfaces $M$ and $N$ whose normal vectors $\overrightarrow{n_{M}}$ and $\overrightarrow{n_{N}}$ are parallel. Thus, we have to find parametrization

$$
x(u(s, t) ; v(s, t))=x(s, t)
$$

and $y(s, t)$ of parts of $M$ and $N$ over a common parameter domain of the $s t$-plane with the property that the normal vectors $\overrightarrow{n_{M}}(s, t)$ and $\overrightarrow{n_{N}}(s, t)$ at $x$ and $y$ are parallel. Let us point out that in case of an arbitrary surface $N$ there is no one-one correspondence between points $x \in M$ and $y \in N$ with $\overrightarrow{n_{M}}(x) \| \overrightarrow{n_{N}}(y)$ (see, [4]). 


\section{Convolution of the surfaces}

Let $M$ be a surface given with the regular patch

$$
M: x(u, v)=\left(u, v, u^{2}+c v^{2}\right), \quad u, v, c \in \mathbb{R}
$$

which is either an elliptic or hyperbolic paraboloid depending on whether $c>0$ or $c<0$. The surface $N$ assumed to admit a local parametrization

$$
N: y(s, t)=\left(y_{1}(s, t), y_{2}(s, t), y_{3}(s, t)\right), \quad s, t \in \mathbb{R},
$$

which is a smooth mapping. The points $x(u, v)=p$ and $y(s, t)=q$ are corresponding if the normal vectors $\overrightarrow{n_{M}}$ and $\overrightarrow{n_{N}}$ at $p$ and $q$, respectively, are linearly dependent. That is;

$$
\overrightarrow{n_{M}}=\lambda \overrightarrow{n_{N}}, \quad 0 \neq \lambda \in \mathbb{R}
$$

Then $p+q$ is a point of the convolution surface $M \star N$ (see, [4]). Assume that the normal vector of $N$ is given with the parametrization of the form

$$
\overrightarrow{n_{N}}=\left(n_{1}(s, t), n_{2}(s, t), n_{3}(s, t)\right)
$$

So, the condition (2.3) gives

$$
\left(\begin{array}{c}
-2 u \\
-2 c v \\
1
\end{array}\right)=\lambda\left(\begin{array}{l}
n_{1}(s, t) \\
n_{2}(s, t) \\
n_{3}(s, t)
\end{array}\right)
$$

In the case of $n_{3}(s, t) \neq 0$ we have

$$
\begin{aligned}
\lambda & =\frac{1}{n_{3}(s, t)}, \\
u(s, t) & =\frac{-n_{1}(s, t)}{2 n_{3}(s, t)}, \\
v(s, t) & =\frac{-n_{2}(s, t)}{2 c n_{3}(s, t)},
\end{aligned}
$$

(see, [4]).

Denoting this reparametrization by the mapping

$$
\phi:(s, t) \rightarrow(u(s, t), v(s, t)),
$$

the surface patch $x(\phi(s, t))$ represents in general the only part of $M$. If the determinant of the Jacobian matrix of $\phi$ does not vanish, then the equation (2.6) represents a regular parametrization. Consequently, we have the following results;

Proposition 2.1. The determinant of the Jacobian matrix of $\phi$ is given by

$$
\operatorname{det}(J \phi)=\frac{1}{4 c n_{3}} \operatorname{det}\left(n_{N}(s, t), n_{N_{s}}(s, t), n_{N_{t}}(s, t)\right) .
$$

Proof. By the definition of the Jacobian matrix

$$
\operatorname{det}(J \phi)=\left|\begin{array}{ll}
\frac{\partial u}{\partial s} & \frac{\partial u}{\partial t} \\
\frac{\partial v}{\partial s} & \frac{\partial v}{\partial t}
\end{array}\right| .
$$

By the use of (2.6), we get

$$
\begin{aligned}
\operatorname{det}(J \phi) & =\left|\begin{array}{ll}
\frac{\left(n_{3}\right)_{s} n_{1}-n_{3}\left(n_{1}\right)_{s}}{2 n_{3}^{2}} & \frac{\left(n_{3}\right)_{t} n_{1}-n_{3}\left(n_{1}\right)_{t}}{2 n_{3}^{2}} \\
\frac{\left(n_{3}\right)_{s} n_{2}-n_{3}\left(n_{2}\right)_{s}}{2 c n_{3}^{2}} & \frac{\left(n_{3}\right)_{t} n_{2}-n_{3}\left(n_{2}\right)_{t}}{2 c n_{3}^{2}}
\end{array}\right| \\
& =\frac{1}{4 c n_{3}} \operatorname{det}\left(n_{N}(s, t), n_{N_{s}}(s, t), n_{N_{t}}(s, t)\right) .
\end{aligned}
$$

This completes the proof of the Proposition 2.1.

Proposition 2.2. The Gaussian curvature of the surface $N$ is given by

$$
\widetilde{K}=\frac{1}{\widetilde{W}^{4}} \operatorname{det}\left(n_{N}(s, t), n_{N_{s}}(s, t), n_{N_{t}}(s, t)\right)
$$

where $\widetilde{W}^{2}=\widetilde{E} \widetilde{G}-\widetilde{F}^{2}$ is the area element of the surface $N$. 
Proof. Let $\widetilde{e}, \widetilde{f}, \widetilde{g}$ be the coefficients of the second fundamental form of the surface $N$

$$
\begin{aligned}
& e^{*}=\left\langle y_{s s}, n_{N}\right\rangle=-\left\langle y_{s},\left(n_{N}\right)_{s}\right\rangle \\
& f^{*}=\left\langle y_{s t}, n_{N}\right\rangle=-\left\langle y_{s},\left(n_{N}\right)_{t}\right\rangle \\
& g^{*}=\left\langle y_{t t}, n_{N}\right\rangle=-\left\langle y_{t},\left(n_{N}\right)_{t}\right\rangle
\end{aligned}
$$

then

$$
\begin{aligned}
e^{*} g^{*}-f^{* 2} & =\left\langle y_{s},\left(n_{N}\right)_{s}\right\rangle\left\langle y_{t},\left(n_{N}\right)_{t}\right\rangle-\left\langle y_{s},\left(n_{N}\right)_{t}\right\rangle\left\langle y_{t},\left(n_{N}\right)_{s}\right\rangle \\
& =\left\langle y_{s} \times y_{t},\left(n_{N}\right)_{s} \times\left(n_{N}\right)_{t}\right\rangle \\
& =\left\langle n_{N},\left(n_{N}\right)_{s} \times\left(n_{N}\right)_{t}\right\rangle(s, t) \\
& =\operatorname{det}\left(n_{N}(s, t),\left(n_{N}\right)_{s}(s, t),\left(n_{N}\right)_{t}(s, t)\right) .
\end{aligned}
$$

Hence,

$$
\begin{aligned}
\widetilde{e} & =\frac{e^{*}}{\widetilde{W}}, \\
\widetilde{f} & =\frac{f^{*}}{\widetilde{W}}, \\
\widetilde{g} & =\frac{g^{*}}{\widetilde{W}},
\end{aligned}
$$

implies that

$$
\begin{aligned}
\widetilde{K} & =\frac{1}{\widetilde{W}^{2}}\left(\widetilde{e} \widetilde{g}-\widetilde{f}^{2}\right) \\
& =\frac{1}{\widetilde{W}^{4}}\left(e^{*} g^{*}-f^{* 2}\right) \\
& =\frac{1}{\widetilde{W}^{4}} \operatorname{det}\left(n_{N}(s, t), n_{N_{s}}(s, t), n_{N_{t}}(s, t)\right) .
\end{aligned}
$$

This completes the proof of the Proposition 2.2.

As a consequence of Proposition 2.1 and Proposition 2.2, we get

Corollary 2.3. The determinant of the Jacobian matrix of the mapping $\phi$ is given by

$$
\begin{aligned}
\operatorname{det}(J \phi) & =\frac{1}{4 c n_{3}} \operatorname{det}\left(n_{N}(s, t), n_{N_{s}}(s, t), n_{N_{t}}(s, t)\right) \\
& =\frac{1}{4 c n_{3}} \widetilde{W}^{4} \widetilde{K} .
\end{aligned}
$$

From Corollary 2.3, it is easy to see that the reparametrization (2.6) is not invertible if $N$ is a developable surface. The final representation of the convolution surface $M \star N$ has the parametrization

$$
(x+y)(s, t)=\left(\frac{-n_{1}(s, t)}{2 n_{3}(s, t)}+y_{1}(s, t), \frac{-n_{2}(s, t)}{2 c n_{3}(s, t)}+y_{2}(s, t), \frac{1}{4 c n_{3}^{2}}\left(c n_{1}^{2}+n_{2}^{2}\right)+y_{3}(s, t)\right) .
$$

The convolution surface $M \star N$ of a paraboloid $M$ and a parametrized surface $N$ consists of the explicit parametrization (2.11).

\section{Some particular surfaces}

In the present section we consider the convolution surface of some special surfaces.

I) Assume that $N$ is a local surface given with the Monge patch

$$
N: y(s, t)=(s, t, h(s, t)),
$$

then the parametrization (2.6) is obtained by

$$
\begin{aligned}
\lambda & =1, \\
u & =\frac{h_{s}}{2}, \\
v & =\frac{h_{t}}{2 c},
\end{aligned}
$$

where $h_{s}$ and $h_{t}$ denote the partial derivatives of $h$ with respect to $s$ and $t$. So, the convolution surface $M \star N$ has the parametrization

$$
(x+y)(s, t)=\left(\frac{h_{s}}{2}+s, \frac{h_{t}}{2 c}+t, \frac{1}{4} h_{s}^{2}+\frac{1}{4 c} h_{t}^{2}+h\right)(s, t) .
$$

If $n_{3}(s, t)=0$, then there exists a curve $\gamma \in N$ such that $\gamma$ is a shadow boundary of $N$. In this case the convolution surface $M \star N$ consists of non-connected parts. 
Definition 3.1. In the Monge patch (3.1), if we take $h(s, t)=f(s)+g(t)$, then the resultant surface given with

$$
N: y(s, t)=(s, t, f(s)+g(t))
$$

is called a translation surface.

We obtain the following result.

Theorem 3.2. Let $M \star N$ be a convolution surface of a paraboloid $M$ and a translation surface $N$ given with the parametrization (3.4). Then the Gaussian curvature of the convolution surface is

$$
K_{M \star N}=\frac{8 c\left(-f^{\prime \prime \prime} f^{\prime}+\left(f^{\prime \prime}\right)^{2}+2 f^{\prime \prime}\right)\left(f^{\prime \prime}+2\right) g^{\prime \prime}}{\left(g^{\prime \prime}+2 c\right)\left(4\left(f^{\prime}\right)^{2}+\left(g^{\prime}\right)^{2}\left(f^{\prime \prime}\right)^{2}+4\left(g^{\prime}\right)^{2} f^{\prime \prime}+4\left(g^{\prime}\right)^{2}+\left(f^{\prime \prime}\right)^{2}+4 f^{\prime \prime}+4\right)^{2}} .
$$

Proof. Let $M \star N$ be a convolution surface of a paraboloid $M$ and a translation surface $N$ given with the parametrization (3.4) For simplicity we define $z=x+y$. Then the tangent space of $M \star N$ is spanned by

$$
\begin{aligned}
& x_{s}=\left(\frac{f^{\prime \prime}}{2}+1,0, f^{\prime}\right) \\
& x_{t}=\left(0, \frac{g^{\prime \prime}}{2 c}+1, \frac{g^{\prime} g^{\prime \prime}}{2 c}+g^{\prime}\right)
\end{aligned}
$$

Hence the coefficients of first and second fundamental forms of the convolution surface $M \star N$ are

$$
\begin{aligned}
& E=\left\langle x_{s}, x_{s}\right\rangle=\left(\frac{f^{\prime \prime}}{2}+1\right)^{2}+\left(f^{\prime}\right)^{2} \\
& F=\left\langle x_{s}, x_{t}\right\rangle=f^{\prime}\left(\frac{g^{\prime} g^{\prime \prime}}{2 c}+g^{\prime}\right) \\
& G=\left\langle x_{t}, x_{t}\right\rangle=\left(\frac{g^{\prime \prime}}{2 c}+1\right)^{2}+\left(\frac{g^{\prime} g^{\prime \prime}}{2 c}+g^{\prime}\right)^{2}
\end{aligned}
$$

and

$$
\begin{aligned}
e & =\frac{\left\langle x_{s s}, x_{s} \times x_{t}\right\rangle}{\sqrt{E G-F^{2}}}=\frac{\left(g^{\prime \prime}+2 c\right)\left(-f^{\prime \prime \prime} f^{\prime}+\left(f^{\prime \prime}\right)^{2}+2 f^{\prime \prime}\right)}{c \sqrt{E G-F^{2}}} \\
f & =\frac{\left\langle x_{s t}, x_{s} \times x_{t}\right\rangle}{\sqrt{E G-F^{2}}}=0, \\
e & =\frac{\left\langle x_{t t}, x_{s} \times x_{t}\right\rangle}{\sqrt{E G-F^{2}}}=\frac{g^{\prime \prime}\left(f^{\prime \prime}+2\right)\left(g^{\prime \prime}+2 c^{2}\right)}{c^{2} \sqrt{E G-F^{2}}}
\end{aligned}
$$

respectively. By definition the Gaussian curvature of the convolution surface $M \star N$ is given by

$$
K_{M \star N}=\frac{e g-f^{2}}{E G-F^{2}}
$$

So, substituting (3.5) and (3.6) into (3.7) we get the result.

Corollary 3.3. Let $M \star N$ be a convolution surface of a paraboloid $M$ and a translation surface (3.4). If the convolution $M \star N$ is a flat surface, then at least one of the following cases occur;

$$
\begin{aligned}
g(t) & =b_{1} t+b_{2}, \\
f(s) & =-s^{2}+d_{1} s+d_{2}, \text { or } \\
f(s) & =\frac{e^{c_{1}\left(s+c_{2}\right)}}{c_{1}^{2}}+\frac{2}{c_{1}} s+c_{2}
\end{aligned}
$$

where $b_{i}, c_{j}, d_{k}$ are real constants.

Proof. If $M \star N$ is a flat surface, then

$$
\left(-f^{\prime \prime \prime}(s) f^{\prime}(s)+\left(f^{\prime \prime}(s)\right)^{2}+2 f^{\prime \prime}(s)\right)\left(f^{\prime \prime}(s)+2\right) g^{\prime \prime}(t)=0
$$

holds. So, we have the three possible cases;

i) $g^{\prime \prime}(t)=0$,

ii) $f^{\prime \prime}(s)+2=0$,

iii) $-f^{\prime \prime \prime}(s) f^{\prime}(s)+\left(f^{\prime \prime}(s)\right)^{2}+2 f^{\prime \prime}(s)=0$.

Solving these differential equations we get the result. This completes the proof of the corollary.

Corollary 3.4. The convolution surface $M \star N$ given with $g(t)=b_{1} t+b_{2}$ is a part of a plane. 
II)Assume that $N$ is a surface of revolution given with the surface patch

$$
N: y(s, t)=(f(s) \cos t, f(s) \sin t, h(s)),
$$

then the equations in (2.6) turns into

$$
\begin{aligned}
\lambda & =\frac{1}{f f^{\prime}} \\
u & =\frac{h^{\prime}}{2 f^{\prime}} \cos t \\
v & =\frac{h^{\prime}}{2 c f^{\prime}} \sin t
\end{aligned}
$$

Finally, convolution surface $M \star N$ has the parametrization

$$
(x+y)(s, t)=\left(\frac{h^{\prime}+2 f f^{\prime}}{2 f^{\prime}} \cos t, \frac{h^{\prime}+2 c f f^{\prime}}{2 c f^{\prime}} \sin t, \frac{h^{\prime}}{2 c\left(f^{\prime}\right)^{2}}\left(c \cos ^{2} t+\sin ^{2} t\right)+h(s)\right) .
$$

Theorem 3.5. Let $M \star N$ be a convolution surface of a paraboloid $M$ and a surface of revolution given with the parametrization (3.8). Then the Gaussian curvature of the convolution surface is

$$
K_{M \star N}=\frac{4 c\left(f^{\prime}\right)^{4} h^{\prime}\left(f^{\prime} h^{\prime \prime}-f^{\prime \prime} h^{\prime}\right)}{\Psi(s, t)} ; f^{\prime} \neq 0 .
$$

where $\Psi(s, t)$ is a real valued non-zero differentiable function of the parameters $s$ and $t$.

Proof. Similar to the proof of Theorem 3.2, we get the result.

Corollary 3.6. Let $M \star N$ be a convolution surface of a paraboloid $M$ and a surface of revolution (3.8). If the convolution surface $M \star N$ is a flat surface, then one of the following cases occur;

i) $N$ is a part of a plane, or

ii) $N$ is a part of a cone, or

iii) $N$ is a part of a paraboloid.

Proof. If $M \star N$ is a flat surface, then

$$
\left(f^{\prime}\right)^{4} h \prime\left(f^{\prime} h^{\prime \prime}-f^{\prime \prime} h^{\prime}\right)=0, f^{\prime} \neq 0
$$

holds. So, we have two possible cases;

i) $h^{\prime}=0$, or

ii) $f^{\prime} h^{\prime \prime}-f^{\prime \prime} h^{\prime}=0$.

If $h^{\prime}=0$ then $N$ is a part of a plane. Further, if $h^{\prime \prime}=0$ and $f^{\prime \prime}=0$ then $N$ is a part of a cone. Finally for the $f^{\prime} h^{\prime \prime}-f^{\prime \prime} h^{\prime}=0$ case with $h^{\prime \prime} \neq 0$ and $f^{\prime \prime} \neq 0$ the surface $N$ is a part of a paraboloid.

III) Assume that $N$ is a conoidal surface given with the parametrization

$$
N: y(s, t)=\left(\begin{array}{c}
p(s) \sin s+p^{\prime}(s) \cos s+t \cos s \\
-p(s) \cos s+p^{\prime}(s) \sin s+t \sin s \\
z(s)
\end{array}\right)
$$

where $p$ and $z$ are real valued differentiable functions. Then, the parametrization (2.6) is obtained by

$$
\begin{aligned}
\lambda & =\frac{-1}{t} \\
u & =\frac{-z^{\prime}(s)}{2 t} \sin s \\
v & =\frac{z^{\prime}(s)}{2 c t} \cos s .
\end{aligned}
$$

Finally, the sum $M \star N$ has the parametrization

$$
(x+y)(s, t)=\left(\begin{array}{c}
\left(\frac{2 t p(s)-z^{\prime}(s)}{2 t}\right) \sin s+\left(p^{\prime}(s)+t\right) \cos s \\
\left(\frac{z^{\prime}(s)-2 c t p(s)}{2 c t}\right) \cos s+\left(p^{\prime}(s)+t\right) \sin s \\
\left(\frac{z^{\prime}(s)^{2}}{4 c t^{2}}\right)\left(c \sin ^{2} s+\cos ^{2} s\right)
\end{array}\right)
$$

If we assume that $p(s)=p$ is a constant function and $z(s)=k s, k \neq 0$ then the conoidal surface $N$ has the parametrization

$$
N: y(s, t)=\left(\begin{array}{c}
p \sin s+t \cos s \\
-p \cos s+t \sin s \\
k s
\end{array}\right)
$$


Consequently, if $p(s)=0$, then the $N$ is a right helicoid

$$
y(s, t)=\left(\begin{array}{c}
t \cos s \\
t \sin s \\
k s
\end{array}\right)
$$

which is a minimal surface. We obtain the following result.

Theorem 3.7. Let $M \star N$ be a convolution surface of a paraboloid $M$ and a right helicoid given with the parametrization (3.17). Then, the Gaussian curvature of the convolution surface is

$$
K_{M \star N}=\frac{4 t^{4} k^{2}}{\left(k^{4}+k^{2} t^{2}-4 k^{2} t^{4}-4 t^{6}\right)\left(k^{2}+t^{2}\right)} .
$$

Proof. Similar to the proof of Theorem 3.2, we get the result.

\section{Visualization}

Example 4.1. For $f(s):=\cos (s), g(t):=\cos (t) ; c:=-3 ;$ we obtain the following graphs of the Monge patch and the convolute surface given the parametrization (3.3);
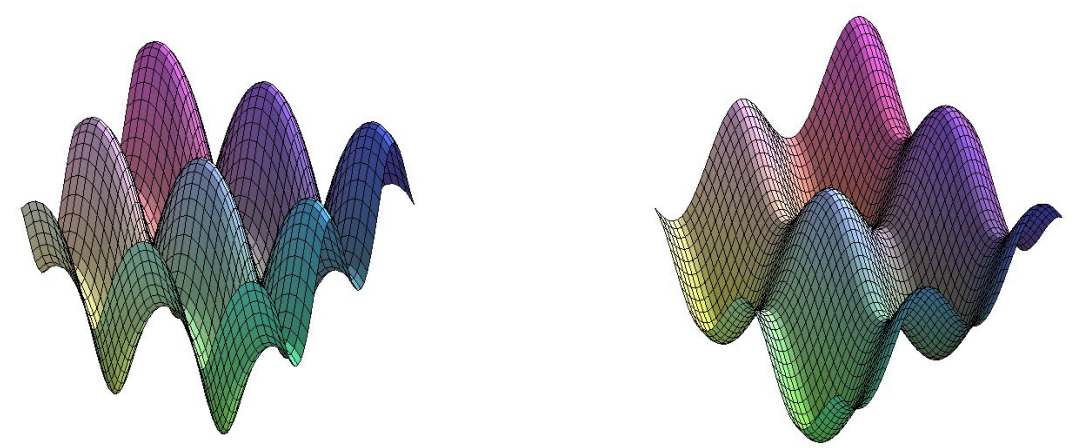

Figure 4.1: Monge patch and its convolute surface

Example 4.2. For $f(s):=s, h(s):=3 s-5 ; c:=-3$; we obtain the following graph of the surface of revolution and the convolute surface given the parametrization (3.10);
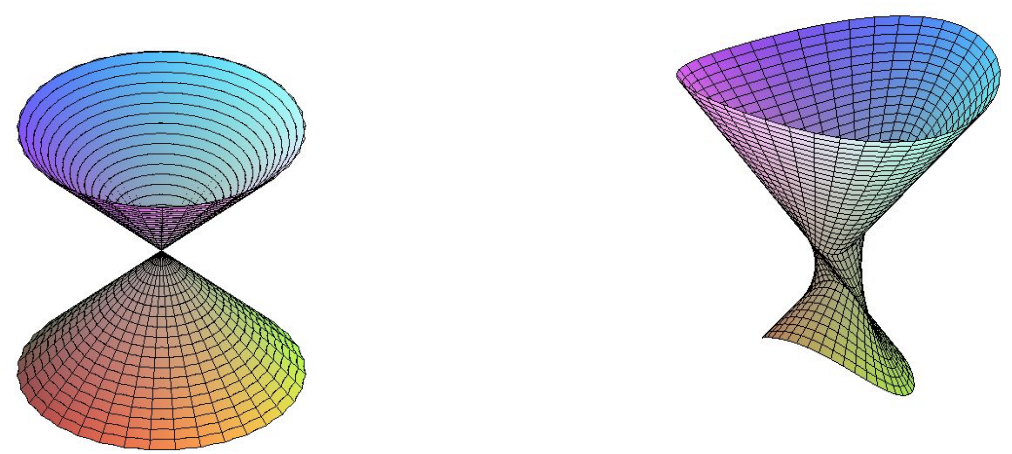

Figure 4.2: Surface of revolution and its convolute surface

Example 4.3. For $p(s):=0, z(s):=2 s$; we obtain the following graph of the conoidal surface and the convolute surface given the parametrization (3.15); 

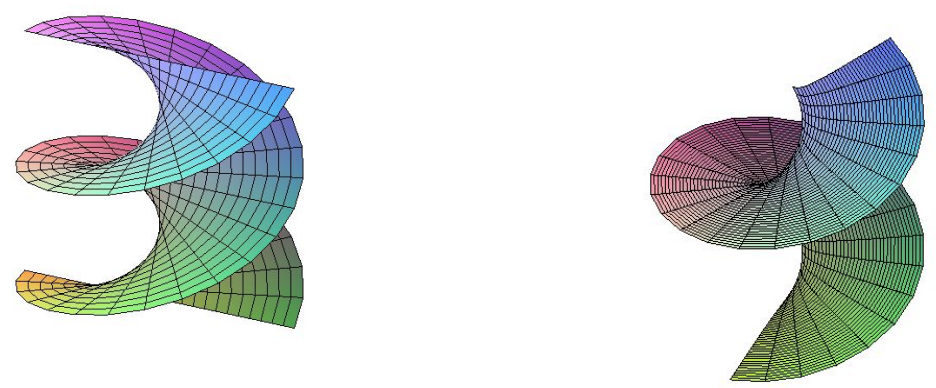

Figure 4.3: The conoidal surface and its convolute surface

\section{Conclusion}

Modelling with curves and surfaces are important area in applied differential geometry. In the present study we consider Minkowski sum of two smooth surfaces in 3-dimensional Euclidean space. This process is also called the convolution of two surfaces. We obtain some nice convolution surfaces by taking some particular surfaces such as Monge patch, surface of revolution and conoidal surfaces. We also plot the graph of the surfaces.

\section{References}

[1] J. Bloomenthal, K. Shoemake, Convolution surfaces, Computer Graphics 25(4) (1991), 251-256.

[2] M. Lavicka, B. Bastl, Z. Sir, Reparameterization of curves and surfaces with respect to convolutions, in: Dæhlen, M., et al.(Eds.), MMCS 2008. In: Lecture Notes in Computer Science, 5862, 2010, 285-298.

[3] M. Peternell, T. Steiner, Minkowski sum boundary surfaces of 3D-objects, Graphical Models 69 (2007), 180-190.

[4] M. Peternell, F. Manhart, The Convolution of a Paraboloid and a Parametrized Surface, www.dmg.tuwien.ac.at/geom/peternell/parsurf_article.pdf

[5] Z. Sir, J. Gravesen, B. Jüttler, Computing Convolutions and Minkowski sums via Support Functions, Industrial Geometry, FSP Report 29, 2006.

[6] J. Vrsek, M. Lavicka, On convolutions of algebraic curves, J. Sym. Comp. 45 (2010), 657-676. 